\title{
RESEARCH ARTICLE \\ Effects of weather and season on human brain volume
}

\author{
Gregory A. Book $\oplus^{1 *}$, Shashwath A. Meda ${ }^{1}$, Ronald Janssen ${ }^{1}$, Alecia D. Dager ${ }^{1,2}$, \\ Andrew Poppe ${ }^{1}$, Michael C. Stevens ${ }^{1,2}$, Michal Assaf ${ }^{1,2}$, David Glahn ${ }^{2,3}$, Godfrey \\ D. Pearlson ${ }^{1,2}$
}

1 Olin Neuropsychiatry Research Center, Hartford Hospital, Hartford, CT, United States of America, 2 Yale University, Department of Psychiatry, New Haven, CT, United States of America, 3 Boston Children's Hospital, Department of Psychiatry, Boston, MA, United States of America

* gregory.a.book@gmail.com

\section{Abstract}

We present an exploratory cross-sectional analysis of the effect of season and weather on Freesurfer-derived brain volumes from a sample of 3,279 healthy individuals collected on two MRI scanners in Hartford, CT, USA over a 15 year period. Weather and seasonal effects were analyzed using a single linear regression model with age, sex, motion, scan sequence, time-of-day, month of the year, and the deviation from average barometric pressure, air temperature, and humidity, as covariates. FDR correction for multiple comparisons was applied to groups of non-overlapping ROIs. Significant negative relationships were found between the left- and right- cerebellum cortex and pressure $(t=-2.25, p=0.049 ; t=-2.771, p=$ $0.017)$. Significant positive relationships were found between left- and right- cerebellum cortex and white matter between the comparisons of January/June and January/September. Significant negative relationships were found between several subcortical ROIs for the summer months compared to January. An opposing effect was observed between the supraand infra-tentorium, with opposite effect directions in winter and summer. Cohen's d effect sizes from monthly comparisons were similar to those reported in recent psychiatric bigdata publications, raising the possibility that seasonal changes and weather may be confounds in large cohort studies. Additionally, changes in brain volume due to natural environmental variation have not been reported before and may have implications for weatherrelated and seasonal ailments.

Copyright: @ 2021 Book et al. This is an open access article distributed under the terms of the Creative Commons Attribution License, which permits unrestricted use, distribution, and reproduction in any medium, provided the original author and source are credited.

Data Availability Statement: All relevant data are within the manuscript and its Supporting Information files.

Funding: The author(s) received no specific funding for this work.

Competing interests: The authors have declared that no competing interests exist.

\section{Introduction}

Several studies testing the effects of weather and season on the human body have found relationships between these environmental factors and incidence or severity of disease. Sales of headache medicines were found to increase when barometric pressure decreases, and spontaneous delivery rates were found to increase when barometric pressure dropped [1,2]. Environmental effects on specific diseases have been studied, including multiple sclerosis (MS), schizophrenia, and Alzheimer's. A significant relationship was found between winter 
conditions and a higher incidence of onset, or recurrence of, multiple sclerosis [3]. A similar relationship was found between MS relapse rates and latitude in which the patient lived, with rates increasing further from the equator [4]. An association has been observed between season and first-break schizophrenia and psychosis, with a stronger effect in males $[5,6]$. Seasonal rhythms in gene expression have been found to be interrupted by Alzheimer's disease [7]. Season and weather associations with symptoms have been investigated from a public health perspective, but the underlying biological response to environmental factors has not been as extensively studied. A study showed an association between hippocampal volume and simulated photoperiod [8]. One study examined the effect of time-of-day on a longitudinal sample of $755 \mathrm{MS}$ and $834 \mathrm{Alz}$ heimer's patients [9], while a second study examined a controlled sample of 19 healthy young adults [10]. Both studies found that total brain volume decreases throughout the day. Studies of cognition have found a seasonal periodicity associated with task performance [11,12].

With a lack of environmental effect studies on biological changes in humans, animal studies can used as a starting point. Human response to environmental rhythms may be similar to that of animals, as animals often alter their physiology to adapt to changing seasonal energy needs. Animal studies have found seasonal structural changes to the hippocampus, total brain volume, and cranium size in mammal, amphibian, and avian species [13-17]. A study of seasonal changes in the brain volume of the common shrew found the cerebellum increasing in volume by $8.0 \%$ from summer to winter and the rest of the brain decreasing in volume by $11.5 \%$ [13]. The tentorium appeared to act as a divider between effect directions in this study, and much larger effects were observed in males.

Change in daylight is a significant factor in seasonal studies, but few studies have taken into account weather conditions, and no studies have examined the effects of weather on brain volume. Weather is often described as temperature, precipitation, and wind speed, but the most significant driver of weather is barometric pressure. Air moves from areas of high pressure to low bringing with it wind, and changes in temperature and precipitation. Unlike temperature and humidity which are well-controlled in MRI scanning environments, pressure is ubiquitous and thus a good weather variable to explore. A phenomenon similar to changes in barometric pressure that has been studied is the effect of high-altitude exposure on brain volume. Highaltitude (HA) exposure has been studied in humans and measurements of brain volume have been conducted. A three month HA exposure caused an increase in brain volume in one study [18]. At sea-level pressure, but in zero-gravity, cosmonauts were found to experience brain volume changes after 189 days in space [19].

Exploring seasonal and weather changes in brain volume is best analyzed using a very large dataset. Using a sample of healthy control subjects collected at the Olin Neuropsychiatry Research Center, located in Hartford, CT USA over a 15 year period, we explored the effects of environmental factors of season and weather on brain volume. Hartford is an ideal location to test weather and seasonal effects because it is near sea-level, experiences four distinct seasons, and a wide range of weather conditions. Because weather is highly correlated with the season, we attempt to separate the effects of weather and season. In addition to exploring weather and seasonal effects, we attempted to replicate previous findings of a diurnal effect on brain volume, and a change in hippocampal volume based on time of year. We additionally compared the effect sizes found in this study to those found in large-scale neuroimaging studies.

\section{Materials and methods}

\section{Imaging data collection \& processing}

Imaging data was gathered retrospectively from approximately 12,600 structural T1-weighted MRI scans collected between August 2003 and October 2018 at the Olin Neuropsychiatry 
Table 1. Six different T1-weighted structural MRI sequences were used across two MRI scanners.

\begin{tabular}{|c|c|c|c|c|c|c|c|}
\hline Scanner & TR (ms) & TE (ms) & TI (ms) & voxel & Bandwidth (Hz) & Notes & Percent \\
\hline Allegra & 2500 & 2.74 & 900 & $1 \times 1 \times 1$ & 190 & & $49.2 \%$ \\
\hline Skyra & 2400 & 2.07 & 1000 & $0.8 \times 0.8 \times 0.8$ & 240 & & $16.7 \%$ \\
\hline Allegra & 2500 & 3.05 & 1000 & $1 \mathrm{x} 1 \mathrm{x} 1$ & 240 & & $15.2 \%$ \\
\hline Skyra & 2300 & 2.91 & 900 & $1 \times 1 \times 1.2$ & 240 & & $9.4 \%$ \\
\hline Skyra & 2200 & 2.88 & 794 & $0.8 \times 0.8 \times 0.8$ & 200 & 5 averages & $6.7 \%$ \\
\hline Skyra & 2200 & 4.13 & 766 & $0.8 \times 0.8 \times 0.8$ & 140 & 4 averages & $2.6 \%$ \\
\hline
\end{tabular}

Research Center, Institute of Living, in Hartford, CT USA. Subjects who received MRI scans were recruited into individual neuropsychiatric studies. Institutional Review Board of Hartford Hospital reviewed and approved the studies under which this data was collected. Those subjects received a complete description of the studies in which they participated and written consent was obtained from research participants. Scans were performed on a Siemens Allegra 3T head-only MRI and a Siemens Skyra 3T MRI scanner (Siemens Medical Solutions, Malvern PA). Six structural T1 MRI pulse sequences were used between the two MRI scanners (Table 1). Images were analyzed automatically using Freesurfer 6.0 [20] and the recon-all command with -all and -notal-check options. Computational analysis was performed using an instance of the Neuroinformatics Database (NiDB) [21] and took 195,000hrs (22.5 years) of CPU time to complete on a 300-core Linux cluster. Subcortical and summary regions of interest were extracted using the default automatic subcortical segmentation (aseg) atlas [22]. Summary ROIs used for analysis included BrainStem, SubCortGrayVol, CortexVol, and CerebralWhiteMatterVol. Lateral ROIs used for analysis included left- and right- amygdala, caudate, cerebellum cortex, cerebellum white matter, hippocampus, pallidum, putamen, thalamus, cerebral white matter, and cerebral cortex. All ROIs were corrected for estimated total intracranial volume (eTIV), to remove effects of head volume.

Recent publications indicate that head motion is associated with a decrease in Freesurfer volumes. To account for possible subject motion, a motion metric was calculated for each subject's dataset using the methods described in the paper by Reuter et al [23]. Motion was estimated from any fMRI timeseries collected in the same imaging study as the T1 with at least 100 time points collected. Timeseries data may have been a task or resting state scan. These motion estimates were calculated by performing rigid realignment using FSL's MCFLIRT tool [24]. The derivative of the resulting motion correction was calculated, giving a displacement value in $\mathrm{mm}$ between adjacent time points, which ignores the effect of slow physical motion in the scanner. Root mean square (RMS) of the maximum displacement in the $\mathrm{x}, \mathrm{y}$, and $\mathrm{z}$-directions were calculated, with the largest value used as a 'motion' variable for later statistical analysis.

After processing of the imaging data through Freesurfer and FSL, cleaning and quality control was performed. Exclusion criteria included: individuals with invalid ages, invalid/ unknown sex, incidental findings (tumor, aneurism, AVM, etc), history of traumatic brain injury, enrollment in pre-surgical mapping studies, and incomplete Freesurfer analyses and/or fMRI data. Arbitrary cutoffs, determined from visual inspection of the data, were used to exclude analyses with outlying results; datasets with a BrainSegVol-to-eTIV (estimated total intracranial volume) ratio of greater than 1.05 or less than 0.6 were excluded, as well as eTIV's less than $900,000 \mathrm{~mm}^{3}$. Due to the size of the remaining sample, hand-editing of Freesurfer segmented surfaces was not performed. However, rendered images of pial surface maps were reviewed and incorrectly segmented subjects were excluded. Thumbnails of raw T1 data were also examined and subjects with visible artifacts (usually motion related) were excluded. For 
subjects with more than one scan, only the most recent MRI scan was included to attempt to balance the sample away from a younger average age. After all quality control and data cleaning, 6,139 subjects remained.

Imaging data was pooled from over 150 separate research projects that primarily studied psychiatric disorders-each with different enrollment criteria, different definitions of healthy, control, and patient, and differing levels of detail for diagnoses. Some individuals received a full structured clinical interview (SCID) to determine DSM diagnosis, but most participants did not undergo formal psychiatric diagnostic interview. Many individuals did not receive a diagnosis but were enrolled in projects that solely enrolled "healthy" participants. Subjects with diagnosis labels of schizophrenia, bipolar, psychosis, major depression, Alzheimer's, traumatic brain injury, and autism were excluded. Subjects who were not explicitly labeled as "healthy" but were enrolled in projects which also enrolled those diagnoses were excluded from analysis. 4,039 subjects explicitly labeled, or implicitly defined as, "healthy controls", remained. The remaining sample included subjects ranging in age from 9-93 years. Subjects younger than 18 were excluded to remove possible developmental effects, and subjects older than 65 were excluded to remove possible aging effects, leaving 3,279 healthy individuals for analysis.

\section{MRI quality control data}

MRI quality control (QC) data was collected semi-regularly over the course of the analysis period. QC MRI scans were collected on the Allegra MRI using an MPRAGE (multiplanar rapid acquisition gradient echo) pulse sequence $(256 \times 240 \times 260$ voxels, $1.3 \times 1 \times 1 \mathrm{~mm}$ voxel size, $2300 \mathrm{~ms}$ TR, $2.91 \mathrm{~ms} \mathrm{TE}$, $9^{\circ}$ flip angle) on an ADNI phantom (The Phantom Laboratory, https://www.phantomlab.com/magphan-adni). QC scans were collected on the Skyra MRI using an MPRAGE sequence (176x240x256 voxels, $1.1 \times 1.1 \times 1.2 \mathrm{~mm}$ voxel size, $2300 \mathrm{~ms}$ TR, $2.95 \mathrm{~ms} \mathrm{TE}, 9^{\circ}$ flip angle) on an ACR small phantom (Newmatic Medical, Caledonia, MI). Signal-to-noise ratio (SNR) was calculated by dividing the signal (mean intensity of non-noise areas) by the noise (mean intensity of the corners of the image volume).

\section{Environmental data}

Weather data was obtained using the National Oceanic and Atmospheric Administration's (NOAA) Local Climatological Data (LCD) search tool for the period of August 4, 2003 to October 30, 2018, from Bradley International Airport, which is the closest weather station with contiguous data for the time period (https://www.ncdc.noaa.gov/cdo-web/datatools/lcd). Bradley Airport is located 12 miles $(20 \mathrm{~km})$ from the MRI collection site and has an elevation of $170 \mathrm{ft}(51.8 \mathrm{~m})$. The Olin Center's elevation is approximately $110 \mathrm{ft}(33.5 \mathrm{~m})$. The LCD dataset contained hourly weather variables used in the analysis: Dry Bulb Temp (temperature in C), Relative Humidity (humidity in \%), Station Pressure (barometric pressure in inHg). The nearest hourly measurement to the start time of the T1 scan was used in analysis. Köppen climate classification identifies Hartford, CT, USA as a humid continental climate (Dfa) characterized by hot summer, cold winter, and well distributed year-round precipitation, with four distinct seasons [25]. For simplicity, astronomical season was defined as starting on the $21^{\text {st }}$ day of March, June, September, and December, so that days of the year 80-171 were labeled spring, days 172-263 labeled summer, days 264-354 labeled fall, and all other days labeled winter. Scan time-of-day was obtained from the DICOM header for the T1 series. Because time-ofyear, temperature, and humidity are highly correlated, we attempted to separate the effects of time of year and weather by using the deviation of weather variables from monthly mean. Mean monthly temperature, pressure, and humidity were calculated over the 15-year period, 
from which the deviation from the monthly averages of weather at individual scan time-points was calculated. This deviation from monthly mean was then used in analysis and referred to as pressure, temperature, and humidity. This method distinguishes the effects of time-of-year from the effects of the departure from normal weather conditions; ie, is an effect of temperature because temperature is hottest in July or because of warmer than average temperatures on any given day of the year.

\section{Statistical analysis}

We wanted to determine if effects seen were due to weather or time-of-year, so a linear model was used where each FreeSurfer ROI served as a dependent measure, and age, motion, sex, time-of-day, and deviation from pressure/temperature/humidity were independent continuous variables, and scan sequence and month were categorical variables. ROIs were selected because they were whole-brain or summary regions (total gray matter, cortex volume, etc) or defined structures (amygdala, putamen, etc) that have been implicated in the limited prior literature. Intracranial volume (ICV) may be change with age, but was not included in the model because of its strong correlation with age. Because of previous evidence of sex differences in brain volume, similar analyses were performed for only males and only females. Analyses were performed using the R statistical software package (http://r-project.org) and significant results with $p<0.05$ were noted, using FDR correction for multiple comparisons across non-overlapping groups of ROIs. Additional post-hoc t-tests were performed for each ROI for a monthmonth comparison. Uncorrected p-values less than 0.05 were noted. Percent difference in volume from mean, and the Cohen's $d$ effect size, of the factor of interest, between months were calculated. For graphical purposes, monthly percent different from annual mean were calculated for each ROI.

Body-mass index data was only available on 517 of the 3279 subjects included in the main analysis. A separate analysis of that subset, using BMI as a covariate was performed, and the results included in supplement Tables 2 and 3 in S1 File.

\section{Results}

\section{Subjects}

Subjects ranged in age from 18 to 65 , with a mean age of $32.4(+/-13.5)$ years; 1,779 female (33.4 +/- 14.2 years), and 1,500 male ( $31.3+/-12.6$ years). Pairwise t-tests by month, FDR corrected for multiple comparisons, showed no significant differences in scan sequence or motion. Significant differences were found in one month-month comparison for sex (supplement Table 1b in S1 File), and eight month-month comparisons for age (supplement Table 1a in S1 File), and no significant differences for motion or scantype by month. MRI quality control data did not indicate an association between phantom SNR and time of year.

\section{Weather}

Weather data was available within +/- two hours for $91.4 \%$ of the scans in the dataset. For the remaining datasets, the nearest weather measurements within six hours were used. Minimum and maximum measurements of pressure, temperature, and humidity during MRI scanning ranged from $-16.1 \mathrm{C}$ to $38.3 \mathrm{C}, 10 \%$ to $100 \%, 28.82 \mathrm{inHg}$ to $30.51 \mathrm{inHg}$ respectively.

\section{Weather effects}

Pressure was negatively associated with supra-tentorial and caudate volumes, while cerebellum cortex and white matter volumes were positively associated with pressure (Table 2). 
Table 2. Effects of weather and time of year (month of scan compared to January) on select ROIs.

\begin{tabular}{|c|c|c|c|c|c|c|c|c|c|c|c|c|c|c|c|}
\hline & \multicolumn{4}{|c|}{ Weather } & \multicolumn{11}{|c|}{ Time of year (Month vs January) } \\
\hline & Pressure & Temp & Humidity & Timeofday & Feb & Mar & Apr & May & Jun & Jul & Aug & Sep & Oct & Nov & Dec \\
\hline BrainSegVol & 1.117 & 0.336 & 0.039 & -0.002 & 0.144 & 0.304 & ${ }^{* *}-2.666$ & -1.125 & -0.578 & -1.542 & -0.832 & 0.939 & 0.490 & -0.797 & -0.684 \\
\hline TotalGrayVol & 0.250 & 0.393 & 0.058 & -0.164 & 0.368 & 1.197 & -1.553 & 0.453 & 0.429 & -0.387 & 0.212 & 1.862 & 1.502 & -0.453 & -0.427 \\
\hline Cerebral WM & 1.786 & -0.166 & 0.113 & 0.597 & 0.136 & -0.604 & ${ }^{* *}-2.769$ & $*-2.234$ & -1.899 & $*-2.413$ & -1.768 & -0.459 & -0.452 & -0.356 & -0.400 \\
\hline CortexVol & 1.449 & 0.063 & -0.389 & -0.078 & 0.784 & 1.118 & -1.711 & 0.102 & -0.435 & -0.891 & -0.295 & 0.939 & 1.167 & -0.358 & 0.410 \\
\hline SubCortGrayVol & 1.152 & 1.465 & -0.275 & 0.086 & -1.335 & 0.222 & ${ }^{* * *}-3.602$ & ${ }^{*}-2.144$ & ${ }^{* *}-3.070$ & ${ }^{*}-2.636$ & ${ }^{* *}-3.377$ & -0.760 & -1.332 & -1.705 & -0.981 \\
\hline L Cerebral WM & 1.707 & -0.077 & 0.218 & 0.538 & 0.049 & -0.590 & ${ }^{*}-2.776$ & $*-2.235$ & -1.839 & -2.263 & -1.753 & -0.334 & -0.400 & -0.275 & -0.297 \\
\hline L CortexVol & 1.303 & 0.265 & -0.217 & -0.073 & 0.604 & 1.101 & -1.795 & 0.045 & -0.388 & -0.907 & -0.378 & 1.014 & 1.131 & -0.436 & 0.385 \\
\hline R Cerebral WM & 1.846 & -0.253 & 0.007 & 0.649 & 0.222 & -0.611 & $*-2.733$ & $*-2.209$ & -1.939 & -2.538 & -1.764 & -0.580 & -0.498 & -0.434 & -0.498 \\
\hline R CortexVol & 1.566 & -0.137 & -0.552 & -0.082 & 0.948 & 1.115 & -1.598 & 0.157 & -0.474 & -0.858 & -0.207 & 0.849 & 1.181 & -0.274 & 0.427 \\
\hline L Lateral Ventricle & 1.006 & 1.007 & -1.069 & -1.547 & -0.983 & -1.004 & -0.642 & $*-2.216$ & 0.051 & 0.360 & -1.156 & -1.416 & -1.759 & -2.304 & -0.389 \\
\hline R Lateral Ventricle & 1.322 & 1.235 & -1.446 & -1.330 & -2.049 & -2.069 & -1.499 & ${ }^{* *}-3.173$ & -1.232 & -0.702 & -1.795 & -2.474 & -2.469 & -2.438 & -1.269 \\
\hline SupraTentorialVol & ${ }^{*} 2.152$ & 0.206 & -0.350 & 0.139 & 0.240 & 0.058 & $*-3.061$ & -1.823 & -1.695 & -2.219 & -1.674 & -0.076 & -0.008 & -0.875 & -0.226 \\
\hline Brain Stem & -1.905 & 1.118 & 1.396 & -0.539 & -0.677 & 0.496 & -0.887 & 0.331 & 0.533 & -0.506 & 0.252 & 0.968 & 0.126 & -0.903 & -1.571 \\
\hline L Cerebellum & ${ }^{*}-2.308$ & 0.369 & 0.863 & -0.397 & -0.275 & 0.608 & 0.821 & 1.587 & ${ }^{* *} 2.802$ & 1.905 & 2.314 & ${ }^{*} 3.092$ & 2.018 & 0.233 & -1.666 \\
\hline L Cerebellum WM & -1.641 & -0.152 & 0.646 & -0.656 & -0.010 & 0.594 & 0.674 & 1.763 & ** 2.999 & 0.999 & 2.395 & $* 2.670$ & 0.951 & 0.648 & -0.442 \\
\hline R Cerebellum & $*-2.807$ & 0.629 & 1.050 & -0.197 & -0.393 & 0.749 & 0.214 & 1.313 & ** 2.496 & 1.222 & 1.543 & * 2.667 & 1.374 & -0.096 & -1.311 \\
\hline R Cerebellum WM & $*-2.592$ & 0.516 & 1.518 & -0.448 & 0.136 & 0.695 & 0.118 & 1.890 & ** 2.819 & 0.926 & 1.942 & * 2.195 & 0.335 & -0.050 & -1.022 \\
\hline L Amygdala & -0.970 & 0.998 & -0.119 & -0.051 & -2.226 & -0.619 & -1.370 & -1.799 & -1.546 & -1.531 & -0.037 & -0.534 & -0.639 & -1.723 & -0.534 \\
\hline L Caudate & 2.525 & -0.501 & -1.449 & -1.233 & -0.662 & 0.139 & ${ }^{* *}-3.167$ & -2.509 & ${ }^{*}-2.711$ & -1.246 & ${ }^{* *}-3.104$ & -0.345 & -1.369 & -2.031 & -1.118 \\
\hline L Hippocampus & 0.343 & 1.288 & 0.934 & 0.719 & -1.268 & 0.117 & -2.074 & -0.395 & -1.516 & -1.216 & -1.369 & 0.530 & 0.106 & -0.407 & -0.494 \\
\hline L Pallidum & 1.093 & 0.722 & -0.385 & -1.043 & -0.890 & -0.306 & -2.043 & -1.749 & ${ }^{* *}-3.528$ & -2.235 & ${ }^{* * *}-4.087$ & -0.737 & -2.010 & -2.772 & 0.037 \\
\hline L Putamen & 0.955 & 1.049 & -0.870 & 0.308 & -0.659 & 0.238 & ${ }^{*}-2.557$ & -1.481 & ${ }^{*}-2.781$ & -2.583 & ${ }^{* *}-3.610$ & -0.784 & -1.295 & -0.659 & -0.905 \\
\hline L Thalamus & 0.592 & 0.829 & 0.317 & 0.979 & -1.431 & 0.287 & ${ }^{* *}-3.078$ & -1.961 & -1.722 & -2.267 & ${ }^{*}-2.542$ & -1.281 & -0.797 & -1.345 & -1.368 \\
\hline R Amygdala & -0.583 & 1.171 & 0.712 & 0.711 & -1.720 & -0.730 & -2.043 & -1.805 & -1.276 & -1.955 & -0.807 & -0.292 & 0.044 & -1.519 & -0.958 \\
\hline R Caudate & 2.694 & -0.673 & -1.253 & -0.444 & -0.127 & 0.497 & -2.005 & -1.523 & ${ }^{*}-2.232$ & -0.635 & -1.934 & 0.133 & -0.780 & -1.883 & -0.622 \\
\hline R Hippocampus & -0.613 & 1.579 & 0.662 & 1.336 & -0.462 & 0.425 & -1.469 & -0.352 & -1.072 & -0.715 & -1.715 & 0.823 & -0.446 & -0.421 & -0.138 \\
\hline R Pallidum & 0.109 & 0.563 & -0.247 & -0.120 & 0.303 & -0.016 & -1.895 & -1.305 & $*-2.760$ & -2.057 & ${ }^{* *}-3.596$ & -1.060 & -1.525 & -2.534 & -0.493 \\
\hline R Putamen & 1.252 & 0.522 & -1.253 & -0.342 & -0.679 & 0.297 & ${ }^{*}-2.595$ & -1.250 & $*-2.653$ & -2.815 & ${ }^{* *}-3.238$ & -1.655 & -1.117 & -0.999 & -0.675 \\
\hline R Thalamus & 0.785 & 1.496 & 0.500 & 0.219 & -1.304 & -0.200 & ${ }^{* *}-3.064$ & -1.894 & ${ }^{*}-2.376$ & -2.325 & ${ }^{* *}-3.308$ & -1.402 & -1.332 & -1.700 & -1.588 \\
\hline
\end{tabular}

*** $p<0.001$.

${ }^{*} p<0.01$.

$* p<0.05$.

Units are t-value, color-coded by size of t-value. Each grouping of ROIs is corrected for multiple comparisons using false discovery rate (FDR) correction method. Pressure, temperature, and humidity are based on the deviation of the weather variable from the monthly mean. Month values are based on comparison between that month and January.

Temperature and humidity were not associated with changes in any brain regions. Effects of pressure were only found in females, and only in the supra-tentorial, left/right cerebellum, and right cerebellum white matter.

\section{Seasonal effects}

Several ROIs showed significant associations with January-June and January-August comparisons (Table 2). More subcortical ROIs were significantly different than January for the month of July in males and August in females (Tables 3 and 4). Females did show significant differences in hippocampal volume between January and August (Table 4). Post-hoc uncorrected ttests by month showed subcortical gray matter volume decreased between January and August $(p=.003$, Cohen's $\mathrm{d}=-.228)$ and increased between August and December $(p=0.013$, Cohen's 
Table 3. Effects of weather and month on brain volume ROIs.

\begin{tabular}{|c|c|c|c|c|c|c|c|c|c|c|c|c|c|c|}
\hline & \multicolumn{3}{|c|}{ Weather } & \multicolumn{11}{|c|}{ Time of year (Month vs January) } \\
\hline & Pressure & Temp & Humidity & Feb & Mar & Apr & May & Jun & Jul & Aug & Sep & Oct & Nov & Dec \\
\hline BrainSegVol & 0.473 & 0.554 & 0.097 & 1.108 & 0.382 & -0.751 & -0.399 & 0.029 & $*-2.317$ & 0.977 & 1.498 & 1.226 & -0.789 & -0.722 \\
\hline TotalGrayVol & 0.266 & 0.183 & 0.491 & 1.085 & 1.677 & 0.697 & 1.113 & 0.826 & -0.525 & *2.108 & $* * 2.629$ & 1.812 & -0.542 & -0.681 \\
\hline Cerebral WM & 0.287 & 0.429 & -0.456 & 0.734 & -1.079 & -2.118 & -1.635 & -1.005 & $* * *-3.746$ & -0.786 & -0.782 & 0.070 & -0.436 & -0.351 \\
\hline CortexVol & 0.692 & -0.277 & 0.153 & 1.149 & 1.23 & -0.087 & 0.156 & 0.235 & -1.009 & 1.194 & 1.822 & 1.272 & -0.473 & 0.113 \\
\hline SubCortGrayVol & 0.960 & 1.643 & -1.164 & -0.595 & 0.183 & -1.915 & -1.759 & -2.395 & $* * *-3.755$ & -1.111 & -1.237 & -1.269 & -1.345 & -0.769 \\
\hline L Cerebral WM & 0.243 & 0.642 & -0.357 & 0.594 & -1.111 & -2.232 & -1.662 & -1.085 & $* *-3.591$ & -0.810 & -0.848 & -0.016 & -0.436 & -0.272 \\
\hline L CortexVol & 0.539 & 0.003 & 0.224 & 0.828 & 1.143 & -0.253 & -0.004 & 0.086 & -0.925 & 1.077 & 1.633 & 0.977 & -0.608 & 0.052 \\
\hline R Cerebral WM & 0.325 & 0.211 & -0.548 & 0.862 & -1.032 & -1.973 & -1.584 & -0.910 & *** -3.842 & -0.750 & -0.704 & 0.155 & -0.429 & -0.423 \\
\hline R CortexVol & 0.822 & -0.541 & 0.08 & 1.429 & 1.281 & 0.077 & 0.307 & 0.373 & -1.062 & 1.276 & 1.956 & 1.523 & -0.329 & 0.168 \\
\hline L Lateral Ventricle & 0.905 & 0.468 & -0.266 & -0.794 & -0.736 & -0.413 & -2.088 & 0.036 & 2.067 & -1.707 & -0.327 & -1.040 & -0.542 & 0.722 \\
\hline $\mathrm{R}$ Lateral Ventricle & 1.358 & 0.457 & -0.825 & -1.272 & -1.660 & -0.561 & $*-3.068$ & -0.770 & 1.650 & -1.550 & -0.957 & -1.406 & -0.75 & 0.643 \\
\hline SupraTentorialVol & 0.880 & 0.350 & -0.464 & 0.986 & -0.216 & -1.767 & -1.604 & -0.811 & ** -3.198 & -0.215 & 0.325 & 0.484 & -0.819 & -0.142 \\
\hline Brain Stem & -0.269 & 1.339 & 1.149 & -0.331 & -0.287 & -0.232 & 0.555 & 0.056 & -1.182 & 1.160 & 0.146 & -0.396 & -1.671 & -1.818 \\
\hline L Cerebellum & -0.611 & 0.503 & 0.949 & 0.581 & 1.600 & 2.384 & $* 2.880$ & 2.242 & 2.100 & ** 3.198 & * 3.092 & 2.187 & 0.242 & -1.230 \\
\hline L Cerebellum WM & -0.490 & 0.602 & 1.24 & 0.202 & 0.580 & 1.875 & 2.100 & 1.256 & 1.154 & 2.260 & $* 2.365$ & 0.969 & 0.054 & -1.010 \\
\hline R Cerebellum & -1.104 & 0.512 & 1.222 & 0.257 & 1.310 & 1.869 & 2.100 & 1.685 & 1.107 & 2.226 & *2.481 & 1.750 & -0.138 & -1.411 \\
\hline R Cerebellum WM & & 0.400 & 1.206 & 0.407 & 0.734 & 1.351 & 2.207 & 1.030 & 0.977 & 2.052 & 1.873 & 1.008 & -0.183 & -1.589 \\
\hline L Amygdala & & & & -2.212 & -0.565 & 0.060 & -1.388 & -1.646 & -1.293 & 0.953 & -0.942 & -1.080 & -1.344 & -1.075 \\
\hline L Caudate & & -0.016 & -0.900 & 0.018 & 0.820 & -0.846 & -2.668 & -1.967 & -0.729 & -1.536 & -0.289 & -1.669 & -0.839 & -0.485 \\
\hline L Hippocampus & 1.196 & 2.175 & -0.199 & -0.265 & 0.419 & -0.041 & 0.406 & -1.163 & -1.570 & 0.862 & -0.174 & -0.097 & 0.288 & 0.028 \\
\hline L Pallidum & 0.966 & 0.393 & -0.399 & 0.171 & -0.149 & -0.811 & -1.150 & -2.053 & ** -3.442 & -1.869 & -0.777 & -1.367 & -1.847 & 0.442 \\
\hline L Putamen & -0.086 & 0.985 & -2.099 & 0.066 & -0.197 & -1.631 & -0.799 & $*-2.667$ & ** -3.509 & -1.959 & -0.791 & -1.271 & -0.502 & -0.910 \\
\hline L Thalamus & 0.779 & 0.877 & -0.947 & -1.320 & -0.323 & -2.603 & -2.305 & -1.556 & $* *-3.604$ & -1.173 & -1.915 & -0.795 & -1.415 & -1.154 \\
\hline R Amygdala & 0.892 & 1.378 & -0.338 & -0.257 & 0.757 & 0.437 & -0.538 & -0.436 & -1.335 & 1.364 & 0.171 & 0.421 & -0.724 & 0.168 \\
\hline R Caudate & 1.641 & -0.114 & -0.625 & 0.280 & 0.652 & -0.376 & -1.953 & -1.739 & -0.344 & -0.928 & -0.185 & -1.073 & -0.864 & -0.108 \\
\hline R Hippocampus & 0.432 & 1.285 & -0.193 & 0.607 & 0.707 & 0.419 & 0.772 & -0.700 & -1.413 & 0.881 & 0.495 & -0.049 & 0.181 & -0.137 \\
\hline R Pallidum & 0.073 & 0.215 & -0.473 & 0.738 & -0.017 & -0.541 & -1.092 & -0.852 & $* * *-3.990$ & -1.471 & -0.554 & -0.447 & -1.325 & 0.382 \\
\hline R Putamen & 0.366 & -0.137 & -1.894 & -0.784 & -0.453 & -2.192 & -1.127 & $*-3.211$ & *** -4.483 & -1.705 & -1.876 & -1.319 & -1.503 & -1.284 \\
\hline R Thalamus & 0.600 & 1.454 & -0.415 & -1.320 & -0.511 & -2.320 & -1.686 & -1.744 & ** -3.261 & -1.511 & -1.748 & -0.930 & -1.633 & -1.141 \\
\hline
\end{tabular}

${ }^{*} p<0.05$

${ }^{* *} p<0.01$.

*** $p<0.001$

Males only $(\mathrm{n}=1500)$.

https://doi.org/10.1371/journal.pone.0236303.t003

$\mathrm{d}=0.203)$. Left- and right- cerebellum cortex increased in volume between January and June ( $p=.003$, Cohen's $\mathrm{d}=.221 ; p=0.011$, Cohen's $\mathrm{d}=0.202)$ and decreased between July and December $(p<.001$, Cohen's $d=-0.262 ; p=0.007$, Cohen's $d=-0.211$. The tentorium acted as a divider between effect direction, with changes from summer to winter months being positive for supra-tentorial ROIs and negative for infra-tentorial ROIs (Table 2). Cerebellum and subcortical volumes showed opposite changes in direction depending on time of year (Fig 1). Cerebellum volumes of male and female showed different trends throughout the year, with peaks occurring in different months (Fig 2).

\section{Effect size comparison}

A comparison of effect sizes was made between this study and those of previously published large-scale studies, using the ENIGMA consortium as an example. ENIGMA has published 
Table 4. Effects of weather and month on brain volume ROIs.

\begin{tabular}{|c|c|c|c|c|c|c|c|c|c|c|c|c|c|c|}
\hline & \multicolumn{3}{|c|}{ Weather } & \multicolumn{11}{|c|}{ Time of year (Month vs January) } \\
\hline & Pressure & Temp & Humidity & Feb & Mar & Apr & May & Jun & Jul & Aug & Sep & Oct & Nov & Dec \\
\hline BrainSegVol & 1.098 & 0.175 & -0.066 & -0.570 & 0.044 & $* *-2.678$ & -1.218 & -0.723 & -0.628 & -1.588 & 0.060 & -0.268 & -0.501 & -0.417 \\
\hline TotalGrayVol & 0.156 & 0.395 & -0.307 & -0.261 & 0.276 & $*-2.273$ & -0.288 & -0.090 & -0.289 & -1.162 & 0.240 & 0.548 & -0.248 & -0.057 \\
\hline Cerebral WM & 2.206 & -0.267 & 0.311 & -0.528 & -0.091 & -2.005 & -1.817 & -1.596 & -0.550 & -1.693 & -0.019 & -0.753 & -0.144 & -0.347 \\
\hline CortexVol & 1.320 & 0.353 & -0.631 & 0.155 & 0.472 & -1.954 & -0.065 & -0.688 & -0.511 & -1.155 & -0.308 & 0.456 & -0.168 & 0.379 \\
\hline SubCortGrayVol & 0.728 & 0.667 & 0.409 & -1.242 & 0.057 & $* *-3.036$ & -1.421 & -2.013 & -0.722 & $* *-3.405$ & -0.015 & -0.795 & -1.096 & -0.721 \\
\hline L Cerebral WM & 2.112 & -0.340 & 0.384 & -0.518 & -0.045 & -1.924 & -1.791 & -1.449 & -0.465 & -1.637 & 0.213 & -0.614 & -0.034 & -0.276 \\
\hline L CortexVol & 1.217 & 0.390 & -0.459 & 0.173 & 0.510 & -1.933 & -0.020 & -0.519 & -0.563 & -1.151 & -0.054 & 0.608 & -0.162 & 0.387 \\
\hline R Cerebral WM & 2.284 & -0.192 & 0.234 & -0.534 & -0.136 & -2.071 & -1.830 & -1.731 & -0.633 & -1.735 & -0.253 & -0.888 & -0.254 & -0.416 \\
\hline R CortexVol & 1.406 & 0.312 & -0.793 & 0.135 & 0.427 & -1.950 & -0.108 & -0.847 & -0.452 & -1.145 & -0.557 & 0.298 & -0.171 & 0.367 \\
\hline L Lateral Ventricle & 0.183 & 0.658 & -0.810 & -0.634 & -0.614 & -0.604 & -0.783 & 0.085 & -1.228 & 0.078 & -1.496 & -1.453 & $*-2.726$ & -1.343 \\
\hline R Lateral Ventricle & 0.221 & 1.049 & -0.883 & -1.651 & -1.162 & -1.587 & -1.082 & -0.968 & -2.273 & -1.021 & -2.431 & -2.093 & $*-2.686$ & -2.517 \\
\hline SupraTentorialVol & ${ }^{*} 2.083$ & 0.197 & -0.211 & -0.433 & 0.106 & -2.544 & -1.303 & -1.471 & -0.849 & -1.863 & -0.392 & -0.449 & -0.562 & -0.279 \\
\hline Brain Stem & $*-2.386$ & 0.136 & 0.894 & -0.491 & 1.049 & -0.791 & 0.145 & 0.747 & 0.311 & -0.564 & 1.159 & 0.658 & 0.314 & -0.414 \\
\hline L Cerebellum & $*-2.575$ & -0.076 & 0.428 & -0.740 & -0.543 & -0.873 & -0.407 & 1.669 & 0.574 & 0.343 & 1.304 & 0.873 & 0.028 & -1.069 \\
\hline L Cerebellum WM & -1.888 & -0.901 & -0.052 & -0.112 & 0.356 & -0.608 & 0.586 & * 2.746 & 0.441 & 1.320 & 1.573 & 0.548 & 0.864 & 0.434 \\
\hline R Cerebellum & $*-2.788$ & 0.329 & 0.348 & -0.626 & -0.116 & -1.247 & -0.068 & 1.759 & 0.523 & 0.161 & 1.237 & 0.452 & -0.069 & -0.424 \\
\hline R Cerebellum WM & $*-2.778$ & 0.217 & 1.137 & -0.113 & 0.299 & -0.938 & 0.658 & $* 2.608$ & 0.405 & 0.863 & 1.322 & -0.253 & 0.125 & 0.143 \\
\hline L Amygdala & -0.834 & -0.884 & -0.245 & -0.926 & -0.318 & -1.691 & -1.182 & -0.564 & -0.921 & -0.736 & 0.153 & 0.042 & -1.035 & 0.263 \\
\hline L Caudate & 1.825 & -0.883 & -1.094 & -0.879 & -0.471 & $*-3.319$ & -0.786 & -1.813 & -1.045 & $*-2.703$ & -0.156 & -0.497 & -1.993 & -1.147 \\
\hline L Hippocampus & -0.510 & 0.121 & 1.128 & -1.358 & -0.275 & $*-2.578$ & -1.021 & -1.038 & -0.532 & $*-2.415$ & 0.880 & 0.054 & -0.687 & -0.668 \\
\hline L Pallidum & 0.550 & 0.399 & -0.104 & -1.317 & -0.306 & -1.960 & -1.269 & $*-2.821$ & -0.323 & $* *-3.666$ & -0.291 & -1.453 & -2.073 & -0.438 \\
\hline L Putamen & 1.432 & 0.691 & 0.536 & -0.994 & 0.265 & -2.052 & -1.650 & -1.537 & -0.793 & $* *-3.028$ & -0.432 & -0.832 & -0.488 & -0.555 \\
\hline L Thalamus & 0.233 & 0.644 & 0.913 & -0.776 & 0.589 & -1.827 & -0.622 & -0.886 & -0.203 & $*-2.410$ & -0.083 & -0.381 & -0.505 & -0.875 \\
\hline R Amygdala & -1.421 & 0.597 & 1.038 & -1.991 & -1.668 & $*-3.000$ & -2.007 & -1.380 & -1.676 & ${ }^{*}-2.227$ & -0.548 & -0.446 & -1.369 & -1.379 \\
\hline R Caudate & 2.019 & -0.865 & -1.218 & -0.415 & 0.123 & -2.234 & -0.174 & -1.408 & -0.585 & -1.722 & 0.336 & -0.201 & -1.769 & -0.817 \\
\hline R Hippocampus & -0.943 & 1.375 & 0.658 & -1.127 & -0.197 & -2.328 & -1.446 & -0.942 & -0.155 & ** -2.919 & 0.619 & -0.733 & -0.713 & -0.067 \\
\hline R Pallidum & 0.185 & 0.657 & -0.075 & -0.326 & -0.118 & -2.152 & -0.846 & $*-2.838$ & 0.266 & $* *-3.498$ & -0.916 & -1.672 & -2.217 & -1.086 \\
\hline R Putamen & 1.311 & 0.745 & -0.004 & -0.289 & 0.540 & -1.683 & -1.059 & -0.911 & -0.317 & $*-2.63$ & -0.585 & -0.440 & -0.097 & 0.039 \\
\hline R Thalamus & 0.575 & 0.838 & 0.881 & -0.596 & 0.165 & -2.020 & -0.990 & -1.622 & -0.567 & $* *-3.129$ & -0.438 & -0.928 & -0.836 & -1.180 \\
\hline $\begin{array}{l}{ }^{*} p<0.05 \\
{ }^{* *} p<0.01 \\
{ }^{* * *} p<0.001 \\
\text { Females only }(\mathrm{n}=17\end{array}$ & & & & & & & & & & & & & & \\
\hline
\end{tabular}

https://doi.org/10.1371/journal.pone.0236303.t004

several large-scale studies comparing Freesurfer derived ROI volumes between controls and patients for various disorders. An analysis of 2,028 schizophrenia patients and 2,540 controls found significant differences in hippocampus, amygdala, thalamus, and lateral ventricles [26]. The effect size of the differences in thalamus volume between populations in the ENIGMA analysis was 0.31 (2.74\% difference), compared to a Cohen's d effect size between March and August in the left- and right- thalamus in the Olin sample of 0.213 (2.98\%) and $0.216(2.94 \%)$. A comparison of 1,728 major depressive disorder (MDD) patients and 7,199 controls showed a significant difference between populations in the hippocampus (1.25\%, Cohen's $d=0.144)$ [27]. March vs August Cohen's $d$ effect sizes for the thalamus in females in this study $(-0.334$ [L]; $-0.329[\mathrm{R}])$ were nearly the same as the effect size for in another paper $(0.358$ [L]; 0.368 [R]) which compared 1,026 epileptics and 1,727 controls [28]. A study of 2,140 substance users 


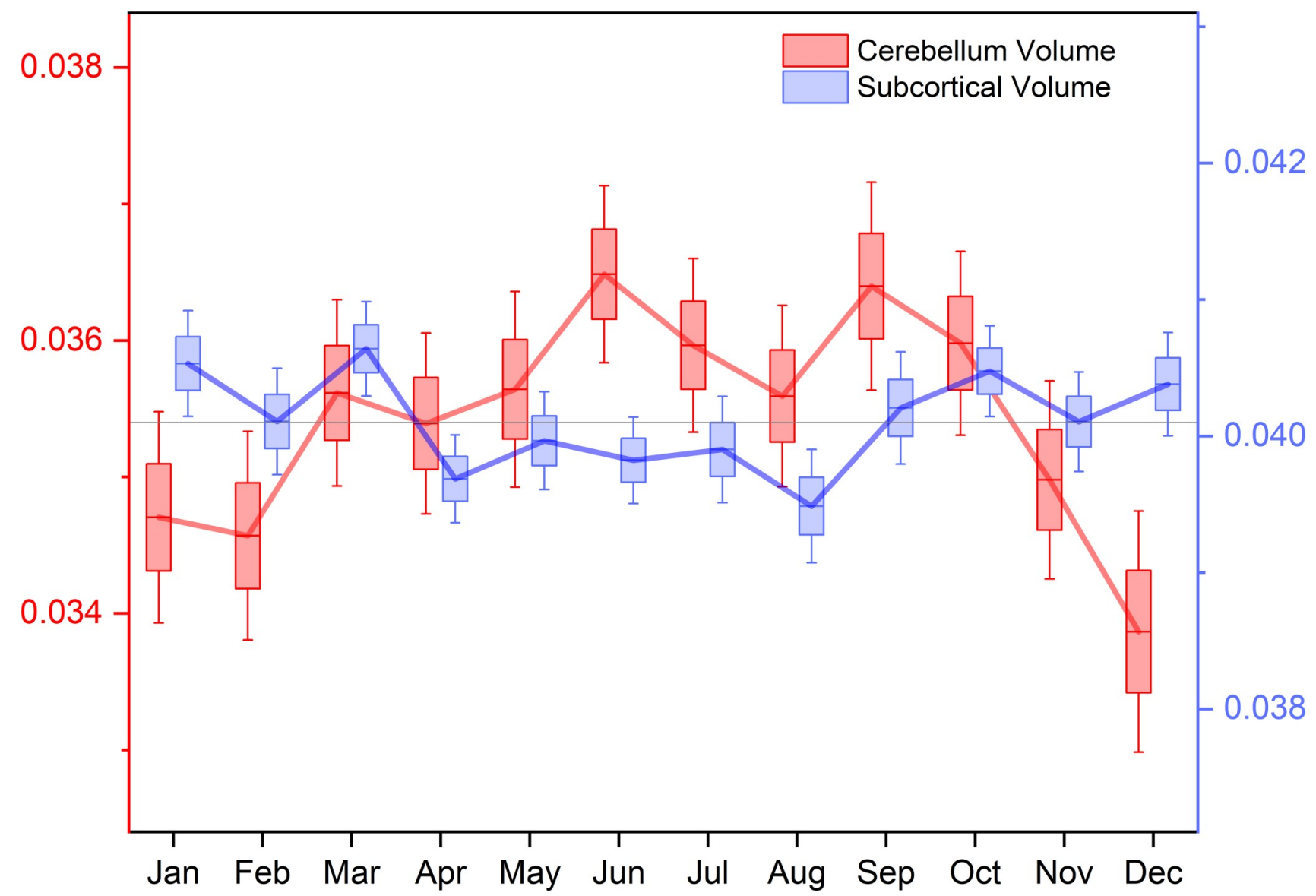

Fig 1. Cerebellum cortex and subcortical volumes compared. Plots aligned to annual mean for each ROI. Box plot indicates standard error, and extension lines indicate $95 \%$ confidence interval. Thick line indicates mean volume.

https://doi.org/10.1371/journal.pone.0236303.g001

and 1,100 controls, compared to this study, found larger effect sizes in this study in the rightamygdala $(0.271>0.111)$, left- and right-hippocampus $(0.296,0.287>0.196,0.180)$, left- and right-putamen $(0.348,-0.358>0.098,0.080)$, right-thalamus $(0.329>0.098)$ [29]. Results from these four papers are listed adjacent to the largest effects found in this study (Table 5).

\section{Discussion}

Season and weather have a known and appreciable effect on the human body, and we have found evidence of previously unmeasured changes in brain volumes. We were unable to replicate a significant time-of-day effect on any brain volume ROI but were able to replicate a seasonal effect on hippocampal volume, though only in females. Time-of-day changes reported in other studies were attributed to hydration status, and we hypothesized that extremes of weather such as a hot dry day, or cool humid day may be reflected in brain volume. No association was observed in our main analysis between time-of-day and brain volume. When controlling for other factors, changes in humidity and temperature from normal had no effect on brain volumes, which suggests that normal hydration status changes may not have an appreciable effect on brain volume. Results from other studies found mixed results on whether hydration status significantly changes the brain volumes measured from MRI images [30,31]. 


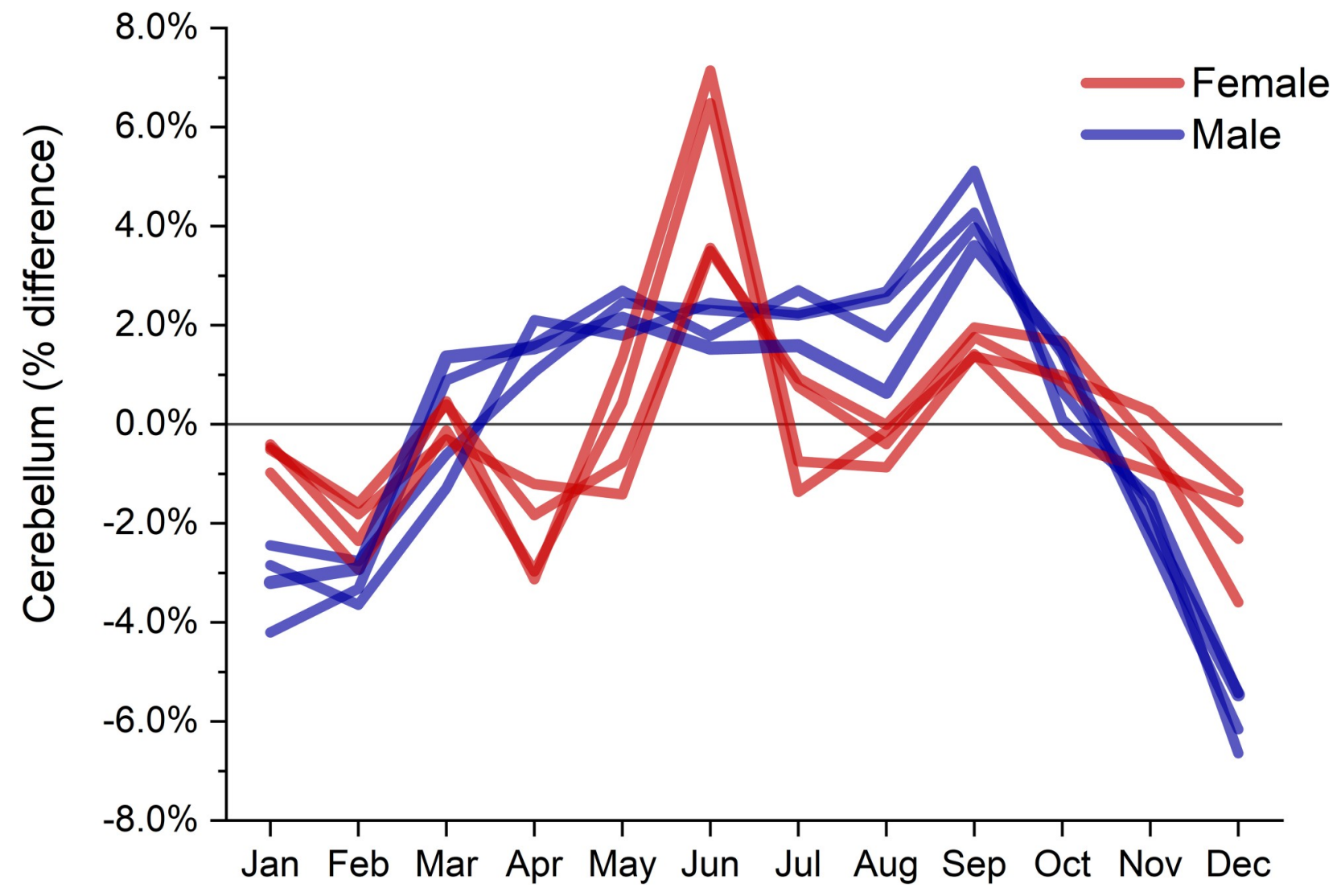

Fig 2. Monthly trends in percent difference from annual mean in left- and right-cerebellum cortex, and left- and right-cerebellum white matter, in males and females. This figure demonstrates the trend peaks at different times of year, but is only for illustrative purposes and not all comparisons between months were statistically significant.

https://doi.org/10.1371/journal.pone.0236303.g002

\section{Biological significance}

Many health effects and diseases are associated with season or weather. Approximately $5 \%$ of the US population experiences seasonal affective disorder in a given year [32]. Headache may have a weather-related trigger as indicated by significantly higher sales of over-the-counter headache medications when barometric pressure dropped the previous day [33]. Studies of the influence of weather on migraine have shown mixed results $[1,34,35]$. Though not neurologically related, drops in barometric pressure cause an increased risk of spontaneous cephalic delivery [2]. Our findings indicate that changes in barometric pressure have a larger effect on the brain volume of females than males, so barometric pressure changes may affect females in multiple ways.

A surprising finding from this study was that supra-tentorial regions gain volume when 'bad' weather approaches-either when barometric pressure drops or when winter is coming -but cerebellum and brain stem volumes change in the opposite direction. While these countervailing effects on different parts of the brain defy easy explanation, they are not without precedent in mammals. Such seasonal brain volume changes are similar to those of the common shrew, with the tentorium acting as a divider between effect directions and with males having larger changes than females. Volume change directions in the shrew are opposite that humans based on time-of-year, however that may be dependent on the average one-and-a-half year lifespan of the shrew. Blood to infra- and supra-tentorial regions are supplied by different 
Table 5. Comparison of effect size of monthly differences from uncorrected t-tests in the Olin sample with effect sizes of previously published large data samples.

\begin{tabular}{|c|c|c|c|c|c|c|c|c|c|}
\hline \multirow[b]{3}{*}{ Freesurfer ROI (aseg atlas) } & \multirow{2}{*}{\multicolumn{3}{|c|}{ Olin sample-largest Cohen's $d^{4}$}} & \multicolumn{6}{|c|}{ ENIGMA } \\
\hline & & & & \multicolumn{2}{|c|}{$\operatorname{MDD}[27]^{1}$} & \multicolumn{2}{|c|}{ SZ [26] ${ }^{1}$} & \multirow{2}{*}{\begin{tabular}{|l|} 
Epilepsy $[28]^{2}$ \\
Cohen's $d$ \\
\end{tabular}} & \multirow{2}{*}{\begin{tabular}{|l} 
Substance $[29]^{3}$ \\
Cohen's $d$ \\
\end{tabular}} \\
\hline & Comparison & $\%$ change & Cohen's $d$ & $\%$ change & Cohen's $d$ & $\%$ change & Cohen's $d$ & & \\
\hline BrainSegVol & Apr $->$ Oct (all) & $1.79 \%$ & ${ }^{*} 0.225$ & & & & & & \\
\hline \multicolumn{10}{|l|}{ BrainStem } \\
\hline SubCortGrayVol & Mat -> Aug (female) & $-4.31 \%$ & $* * *-0.370$ & & & & & & \\
\hline SupratenorialVol & Feb -> Jul (male) & $-2.08 \%$ & ${ }^{*}-0.344$ & & & & & & \\
\hline CortexVol & Aug $->$ Oct (all) & $2.23 \%$ & ${ }^{*} 0.213$ & & & & & & \\
\hline L CortexVol & Aug -> Oct (female) & $3.04 \%$ & $* 0.282$ & & & & & & \\
\hline R CortexVol & Aug $->$ Oct (all) & $2.16 \%$ & ${ }^{*} 0.205$ & & & & & & \\
\hline CerebralWhiteMatterVol & Feb -> Jul (male) & $-3.81 \%$ & ** -0.388 & & & & & & \\
\hline L CerebralWhiteMatterVol & Feb -> Jul (male) & $-3.53 \%$ & ${ }^{*}-0.362$ & & & & & & \\
\hline R CerebralWhiteMatterVol & Feb -> Jul (male) & $-4.08 \%$ & ** -0.404 & & & & & & \\
\hline L Amygdala & & & & $0.66 \%$ & 0.060 & $3.80 \%$ & $0.31 \dagger$ & 0.327 & 0.107 \\
\hline L Caudate & Mar $->$ Aug (female) & $-5.02 \%$ & * -0.305 & $0.23 \%$ & 0.023 & $0.20 \%$ & 0.02 & & \\
\hline L CerebellumCortex & Sep -> Dec (male) & $-9.75 \%$ & $* *-0.372$ & & & & & & \\
\hline L CerebellumWhiteMatter & Apr -> Jun (female) & $9.75 \%$ & ${ }^{*} 0.303$ & & & & & & \\
\hline L Hippocampus & Aug -> Oct (female) & $4.39 \%$ & ${ }^{*} 0.296$ & $1.25 \%$ & $0.144 \dagger$ & $4.10 \%$ & $0.46 \dagger$ & 0.353 & 0.196 \\
\hline L LateralVentricle & May -> Jul (male) & $29.76 \%$ & ${ }^{*} 0.296$ & $1.35 \%$ & 0.056 & $18.24 \%$ & $0.37 \dagger$ & 0.288 & \\
\hline L Pallidum & Jul -> Dec (male) & $5.62 \%$ & ${ }^{* *} 0.357$ & $0.05 \%$ & 0.001 & $2.28 \%$ & 0.21 & & \\
\hline L Putamen & Mar $->$ Aug (female) & $-6.72 \%$ & ${ }^{* * *}-0.348$ & $0.10 \%$ & 0.012 & $0.20 \%$ & 0.08 & & 0.098 \\
\hline L ThalamusProper & Mar $->$ Aug (female) & $-4.46 \%$ & ** -0.334 & $0.40 \%$ & 0.044 & $2.74 \%$ & $0.31 \dagger$ & 0.358 & \\
\hline R Amygdala & Jan -> Apr (female) & $-4.47 \%$ & ${ }^{*}-0.271$ & $0.66 \%$ & 0.060 & $3.80 \%$ & $0.31 \dagger$ & 0.218 & 0.111 \\
\hline R Caudate & Mar -> Aug (all) & $-3.55 \%$ & ${ }^{*}-0.211$ & $0.23 \%$ & 0.023 & $0.20 \%$ & 0.02 & & \\
\hline R CerebellumCortex & Sep -> Dec (male) & $-8.74 \%$ & ** -0.344 & & & & & & \\
\hline R CerebellumWhiteMatter & Apr -> Jun (female) & $10.64 \%$ & ${ }^{*} 0.314$ & & & & & & \\
\hline R Hippocampus & Aug -> Dec (female) & $4.44 \%$ & ${ }^{*} 0.287$ & $1.25 \%$ & $0.144 \dagger$ & $4.10 \%$ & $0.46 \dagger$ & 0.336 & 0.180 \\
\hline R LateralVentricle & May -> Jul (male) & $32.66 \%$ & ** 0.357 & $1.35 \%$ & 0.056 & $18.24 \%$ & $0.37 \dagger$ & 0.268 & \\
\hline $\mathrm{R}$ Pallidum & Feb -> Jul (male) & $-6.47 \%$ & ${ }^{* * *}-0.446$ & $0.05 \%$ & 0.001 & $2.28 \%$ & 0.21 & 0.316 & \\
\hline R Putamen & Jan -> Jul (male) & $-5.65 \%$ & ${ }^{* *}-0.358$ & $0.10 \%$ & 0.012 & $0.20 \%$ & 0.08 & & 0.080 \\
\hline R ThalamusProper & Mar $->$ Aug (female) & $-4.34 \%$ & ${ }^{* *}-0.329$ & $0.40 \%$ & 0.044 & $2.74 \%$ & $0.31 \dagger$ & 0.368 & 0.098 \\
\hline
\end{tabular}

${ }^{*} p<0.05$.

${ }^{* *} p<0.01$.

*** $p<0.001$.

$\dagger$-significance at least $p<0.05$.

1 -Values reported in paper were combined left and right. Values listed in this table are the absolute value of the original value, and repeated in the Left- and Right- ROI rows for comparison.

2 -Cohen's $d$ values listed in this table are the absolute values of the original value reported in the ENIGMA epilepsy paper, and are taken from the "all epilepsies" group comparisons.

3 -Absolute values of original paper. Only values with significance of $p<0.05$ were listed in original paper.

4 -For comparison of effect size between Olin sample and other papers-the largest Cohen's $d$ value and corresponding \% change with the groups compared that was significant at $p<0.05$ uncorrected.

The absolute value is listed for the effect size and percent change of the ENIGMA analyses. ENIGMA analyses that reported combined left and right subcortical structure volumes are repeated next to the left- and right- subcortical structures of the Olin sample. Gray boxes indicate the largest effect size reported for that ROI. Boxes with a border indicate the largest percent difference for that ROI.

vasculature, which may be responsible for opposite changes in volumes. A strong seasonality effect raises a possible explanation of changing vitamin-D levels. Previous studies have found negative association between vitamin-D levels and intracranial volume, and vitamin-D and 
season. Subjects with lower levels of vitamin-D showed larger intracranial and white-matter volumes [36], and lower levels of vitamin-D are found in winter [37]. Though this hypothesis is speculative, it is testable in subjects who are prescribed light exposure during winter months for various conditions.

A possible explanation of brain volume changes is from a change in blood flow as previous studies have found a seasonal effect on ambulatory blood pressure [38,39]. Blood flow associated with barometric pressure may also offer an explanation. The decrease in barometric pressure associated with an increase in infra-tentorial volumes found in this study may be explained by a vascular response to available oxygen levels. Oxygen concentration in the atmosphere in a low-pressure weather system (28.5inHg at sea level) is similar to the oxygen levels (97\% of normal) found at an elevation of $400 \mathrm{~m}$ above sea level. Lower blood oxygen concentration $\left(\mathrm{SpO}_{2}\right)$ is associated with lower barometric pressure [40]. An imaging study of mice subjected to low levels of $\mathrm{O}_{2}$ found that macro-vasculature decreased in volume, while microvasculature blood flow increased [41]. Tissue requires more blood to deliver the same amount of oxygen in a low- $\mathrm{O}_{2}$ environment and may thus cause a small and temporary change in brain volume. However, this does not explain why the cerebellum follows a different pattern from the rest of the brain. Differences in effect direction between the surpra- and intra-tentorium may be due to the blood vessels supplying those two regions of the brain, in that the cerebellum and brain stem have a different blood supply than the cerebrum [42].

Since brain volumes have been assumed to be static except for the effects of aging, few if any studies have examined temporally fine-grained (daily or weekly) MRI scans for extended periods of time. Replication of the changes found in this analysis would be best tested using a single subject, or set of subjects, scanned daily throughout an entire year. Such data would confirm whether the effects seen in this study are a biological effect or are only found at a grouplevel in a heterogeneous group. Either finding would be important to the interpretation of large-scale heterogeneous studies.

Investigating the biological cause of such large volume changes may be clinically relevant, including why volume changes are observed in opposite directions in the supratentorium vs infratentorium. Investigating these changes further may be informative for seasonal disorders or discover previously unknown seasonal effects on other diseases. From a purely statistical standpoint, adding season and weather variables to big data analyses may improve accuracy, especially if the analysis includes geographic sites that experience wide variation in these variables.

\section{Environmental factors as confounds}

A secondary aim of this study was to compare the effect sizes between environmental factors and other big-data papers to see if environmental factors may need to be considered as confounds when performing large cross-site analyses. Many studies have begun aggregating large samples from psychiatric patients to look for evidence that specific psychiatric diagnoses might have different brain structure than non-patient samples [43]. The effect sizes of brain volume changes observed in this study reveal a possible confound in this approach to big data analysis, as those effect sizes were in some cases larger than the effect sizes of patient/control comparisons in recent big-data analyses. This potentially represents a considerable confound when drawing conclusions about patient/control population if these other sources of variation are not controlled. It has been shown that changes in environmental temperature and barometric pressure are known to affect blood pressure and oxygen saturation, and are considered confounds to accurate vital sign measurement in clinical environments [40,44]. Our 
comparisons indicate that such confounds from barometric pressure and season may also exist in neuroimaging studies.

When performing large sample analyses, many unknown factors may influence results. The current results suggest that data collection should be uniform across season, but also that it should be standard practice to statistically model for variation due to season in geographical areas where seasons are distinct and widely variable. It is likely important to include barometric pressure as a statistical covariate when using data gathered from climatologically diverse data collection sites. It is entirely possible that a case-control research study recruits most of the patients at the start of the project in the winter and fills in the controls the following summer. Scanning more subjects of one group in a season might represent the effect seen in a case-control analysis, especially as the effects observed in these analyses are already somewhat small.

\section{Limitations}

Many demographic and phenotypic variables were not collected for all subjects used in this analysis. Variables such as race, ethnicity, education, BMI, medication, menstrual cycle, recreational drug use, and smoking status were only collected on a small subset of subjects, and those subsets often did not overlap, were inconsistently recorded between projects, or were mostly just not available because of inaccessibility to paper records.

\section{Supporting information}

\section{S1 Data.}

(CSV)

S2 Data.

(TXT)

S1 File.

(DOCX)

\section{Acknowledgments}

We would like to acknowledge the entire past and present staff of the Olin Neuropsychiatry Research Center for 15 years' worth of MRI data collection and the individual projects under which the data was collected.

\section{Author Contributions}

Conceptualization: Gregory A. Book, Michael C. Stevens, David Glahn.

Data curation: Gregory A. Book.

Formal analysis: Gregory A. Book, Alecia D. Dager, Andrew Poppe, Michael C. Stevens.

Funding acquisition: Michael C. Stevens, Michal Assaf, David Glahn, Godfrey D. Pearlson.

Investigation: Gregory A. Book.

Methodology: Gregory A. Book, Shashwath A. Meda, Ronald Janssen, Alecia D. Dager, Andrew Poppe, David Glahn.

Project administration: Gregory A. Book.

Supervision: Gregory A. Book. 
Validation: Gregory A. Book, Andrew Poppe, Godfrey D. Pearlson.

Visualization: Gregory A. Book.

Writing - original draft: Gregory A. Book.

Writing - review \& editing: Gregory A. Book, Shashwath A. Meda, Ronald Janssen, Alecia D. Dager, Michael C. Stevens, Michal Assaf, Godfrey D. Pearlson.

\section{References}

1. Hoffmann J., et al., The influence of weather on migraine-are migraine attacks predictable? Ann Clin Transl Neurol, 2015. 2(1): p. 22-8. https://doi.org/10.1002/acn3.139 PMID: 25642431

2. Akutagawa O., Nishi H., and Isaka K., Spontaneous delivery is related to barometric pressure. Arch Gynecol Obstet, 2007. 275(4): p. 249-54. https://doi.org/10.1007/s00404-006-0259-3 PMID: 17004080

3. Ma J. and Zhang X., [The relationship between season/latitude and multiple sclerosis]. Zhonghua Nei Ke Za Zhi, 2015. 54(11): p. 945-8. PMID: 26759213

4. Spelman T., et al., Seasonal variation of relapse rate in multiple sclerosis is latitude dependent. Ann Neurol, 2014. 76(6): p. 880-90. https://doi.org/10.1002/ana.24287 PMID: 25283272

5. Hallam K.T., et al., Seasonal influences on first-episode admission in affective and non-affective psychosis. Acta Neuropsychiatr, 2006. 18(3-4): p. 154-61. https://doi.org/10.1111/j.1601-5215.2006. 00147.x PMID: 26989967

6. Owens N. and McGorry P.D., Seasonality of symptom onset in first-episode schizophrenia. Psychol Med, 2003. 33(1): p. 163-7. https://doi.org/10.1017/s0033291702006712 PMID: 12537047

7. Lim A.S., et al., Diurnal and seasonal molecular rhythms in human neocortex and their relation to Alzheimer's disease. Nat Commun, 2017. 8: p. 14931. https://doi.org/10.1038/ncomms14931 PMID: 28368004

8. Miller M.A., et al., Photoperiod is associated with hippocampal volume in a large community sample. Hippocampus, 2015. 25(4): p. 534-43. https://doi.org/10.1002/hipo.22390 PMID: 25394737

9. Nakamura K., et al., Diurnal fluctuations in brain volume: Statistical analyses of MRI from large populations. Neuroimage, 2015. 118: p. 126-32. https://doi.org/10.1016/j.neuroimage.2015.05.077 PMID: 26049148

10. Trefler A., et al., Impact of time-of-day on brain morphometric measures derived from T1-weighted magnetic resonance imaging. Neuroimage, 2016. 133: p. 41-52. https://doi.org/10.1016/j.neuroimage. 2016.02.034 PMID: 26921714

11. Meyer C., et al., Seasonality in human cognitive brain responses. Proc Natl Acad Sci U S A, 2016. 113 (11): p. 3066-71. https://doi.org/10.1073/pnas.1518129113 PMID: 26858432

12. Lim A.S.P., et al., Seasonal plasticity of cognition and related biological measures in adults with and without Alzheimer disease: Analysis of multiple cohorts. PLoS Med, 2018. 15(9): p. e1002647. https:// doi.org/10.1371/journal.pmed.1002647 PMID: 30180184

13. Lazaro J., et al., Profound seasonal changes in brain size and architecture in the common shrew. Brain Struct Funct, 2018. 223(6): p. 2823-2840. https://doi.org/10.1007/s00429-018-1666-5 PMID: 29663134

14. Luo Y., et al., Seasonality and brain size are negatively associated in frogs: evidence for the expensive brain framework. Sci Rep, 2017. 7(1): p. 16629. https://doi.org/10.1038/s41598-017-16921-1 PMID: 29192284

15. Clayton N.S., Reboreda J.C., and Kacelnik A., Seasonal changes of hippocampus volume in parasitic cowbirds. Behav Processes, 1997. 41(3): p. 237-43. https://doi.org/10.1016/s0376-6357(97)00050-8 PMID: 24896856

16. Edwards F.A. and Gage P.W., Seasonal changes in inhibitory currents in rat hippocampus. Neurosci Lett, 1988. 84(3): p. 266-70. https://doi.org/10.1016/0304-3940(88)90518-6 PMID: 3352952

17. laskin V.A., [Seasonal changes in hippocampus size and spatial behaviour in mammals and birds]. Zh Obshch Biol, 2011. 72(1): p. 27-39. PMID: 21469347

18. Fan C., et al., Reversible Brain Abnormalities in People Without Signs of Mountain Sickness During High-Altitude Exposure. Sci Rep, 2016. 6: p. 33596. https://doi.org/10.1038/srep33596 PMID: 27633944

19. Van Ombergen A., et al., Brain Tissue-Volume Changes in Cosmonauts. N Engl J Med, 2018. 379(17): p. 1678-1680. https://doi.org/10.1056/NEJMc1809011 PMID: 30354959 
20. Fischl B., FreeSurfer. Neuroimage, 2012. 62(2): p. 774-81. https://doi.org/10.1016/j.neuroimage.2012. 01.021 PMID: 22248573

21. Book G.A., et al., Neuroinformatics Database (NiDB) - a modular, portable database for the storage, analysis, and sharing of neuroimaging data. Neuroinformatics, 2013. 11(4): p. 495-505. https://doi.org/ 10.1007/s12021-013-9194-1 PMID: 23912507

22. Fischl B., et al., Whole brain segmentation: automated labeling of neuroanatomical structures in the human brain. Neuron, 2002. 33(3): p. 341-55. https://doi.org/10.1016/s0896-6273(02)00569-x PMID: 11832223

23. Reuter M., et al., Head motion during MRI acquisition reduces gray matter volume and thickness estimates. Neuroimage, 2015. 107: p. 107-115. https://doi.org/10.1016/j.neuroimage.2014.12.006 PMID: 25498430

24. Jenkinson M., et al., Improved optimization for the robust and accurate linear registration and motion correction of brain images. Neuroimage, 2002. 17(2): p. 825-41. https://doi.org/10.1016/s1053-8119 (02)91132-8 PMID: 12377157

25. Peel M. C., B.L.F., McMahon T. A., Updated world map of the Koppen-Geiger climate classification. Hydrology and Earth Science Systems, 2007. 11: p. 1633-1644.

26. van Erp T.G., et al., Subcortical brain volume abnormalities in 2028 individuals with schizophrenia and 2540 healthy controls via the ENIGMA consortium. Mol Psychiatry, 2016. 21(4): p. 547-53. https://doi. org/10.1038/mp.2015.63 PMID: 26033243

27. Schmaal L., et al., Subcortical brain alterations in major depressive disorder: findings from the ENIGMA Major Depressive Disorder working group. Mol Psychiatry, 2016. 21(6): p. 806-12. https://doi.org/10. 1038/mp.2015.69 PMID: 26122586

28. Whelan C.D., et al., Structural brain abnormalities in the common epilepsies assessed in a worldwide ENIGMA study. Brain, 2018. 141(2): p. 391-408. https://doi.org/10.1093/brain/awx341 PMID: 29365066

29. Mackey S., et al., Mega-Analysis of Gray Matter Volume in Substance Dependence: General and Substance-Specific Regional Effects. Am J Psychiatry, 2018: p. appiajp201817040415. https://doi.org/10. 1176/appi.ajp.2018.17040415 PMID: 30336705

30. Meyers S.M., et al., Does hydration status affect MRI measures of brain volume or water content? J Magn Reson Imaging, 2016. 44(2): p. 296-304. https://doi.org/10.1002/jmri.25168 PMID: 26825048

31. Streitburger D.P., et al., Investigating structural brain changes of dehydration using voxel-based morphometry. PLoS One, 2012. 7(8): p. e44195. https://doi.org/10.1371/journal.pone.0044195 PMID: 22952926

32. Kurlansik S.L. and Ibay A.D., Seasonal affective disorder. Am Fam Physician, 2012. 86(11): p. 103741. PMID: 23198671

33. Ozeki K., et al., Weather and headache onset: a large-scale study of headache medicine purchases. Int J Biometeorol, 2015. 59(4): p. 447-51. https://doi.org/10.1007/s00484-014-0859-8 PMID: 24943052

34. Cioffi l., et al., Effect of weather on temporal pain patterns in patients with temporomandibular disorders and migraine. J Oral Rehabil, 2017. 44(5): p. 333-339. https://doi.org/10.1111/joor.12498 PMID: 28244179

35. Becker W.J., Weather and migraine: can so many patients be wrong? Cephalalgia, 2011. 31(4): p. 387-90. https://doi.org/10.1177/0333102410385583 PMID: 21163817

36. Annweiler C., et al., Vitamin D-related changes in intracranial volume in older adults: a quantitative neuroimaging study. Maturitas, 2015. 80(3): p. 312-7. https://doi.org/10.1016/j.maturitas.2014.12.011 PMID: 25614220

37. Klingberg E., et al., Seasonal variations in serum 25-hydroxy vitamin D levels in a Swedish cohort. Endocrine, 2015. 49(3): p. 800-8. https://doi.org/10.1007/s12020-015-0548-3 PMID: 25681052

38. Kristal-Boneh E., et al., Summer-winter variation in $24 \mathrm{~h}$ ambulatory blood pressure. Blood Press Monit, 1996. 1(2): p. 87-94. PMID: 10226208

39. Stergiou G.S., et al., Seasonal variation in meteorological parameters and office, ambulatory and home blood pressure: predicting factors and clinical implications. Hypertens Res, 2015. 38(12): p. 869-75. https://doi.org/10.1038/hr.2015.96 PMID: 26333360

40. Pope C.A.r., et al., Oxygen saturation, pulse rate, and particulate air pollution: A daily time-series panel study. Am J Respir Crit Care Med, 1999. 159(2): p. 365-72. https://doi.org/10.1164/ajrccm.159.2. 9702103 PMID: 9927345

41. Jia Y., et al., Responses of peripheral blood flow to acute hypoxia and hyperoxia as measured by optical microangiography. PLoS One, 2011. 6(10): p. e26802. https://doi.org/10.1371/journal.pone.0026802 PMID: 22046363 
42. Jimsheleishvili S, Dididze M. Neuroanatomy, Cerebellum. [Updated 2020 Jul 31]. In: StatPearls [Internet]. Treasure Island (FL): StatPearls Publishing; 2020 Jan-. Available from: https://www.ncbi.nlm.nih. gov/books/NBK538167/

43. Fan J., Han F., and Liu H., Challenges of Big Data Analysis. Natl Sci Rev, 2014. 1(2): p. 293-314.

44. Jehn M., et al., The effect of ambient temperature and barometric pressure on ambulatory blood pressure variability. Am J Hypertens, 2002. 15(11): p. 941-5. https://doi.org/10.1016/s0895-7061(02) 02999-0 PMID: 12441212 